\title{
Preserved para-arteriole retinal pigment epithelium (PPRPE) in retinitis pigmentosa
}

\author{
JOHN R. HECKENLIVELY \\ From the Jules Stein Eye Institute and the Department of Ophthalmology, UCLA School of Medicine, \\ Los Angeles, California, 90024, USA
}

SUMMARY Five patients with retinitis pigmentosa (RP) with probable autosomal recessive inheritance have been identified in whom there is relative preservation of retinal pigment epithelium adjacent to and under retinal arterioles despite a panretinal degenerative process. All the patients were hypermetropic, though patients with RP tend to be myopic. This implies that there is a factor associated with retinal arterioles which locally retards the RP process in these patients. It may be appropriate to look for the PPRPE pattern in hypermetropic RP patients.

Because more specific diagnostic tests generally are not available, many retinal dystrophies have been named on the basis of distinctive retinal patterns. This has resulted in such interesting names as vitelliruptive macular dystrophy, ${ }^{1}$ butterfly-shaped pigment dystrophy, ${ }^{2}$ fenestrated sheen macular dystrophy, ${ }^{3}$ and reticular dystrophy. ${ }^{4}$ Morphological fundus patterns have also been used in referring to unusual forms of retinitis pigmentosa (RP). These include pigmented paravenous retinochoroidal atrophy, ${ }^{5}$ helicoid parapapillary chorioretinal degeneration, ${ }^{6}$ sector retinitis pigmentosa, ${ }^{7}$ retinitis punctata albescens, ${ }^{8}$ and retinitis pigmentosa sine pigmento.?

Morphological classification of the pigmented retinopathies is a convenient method for separating the various forms of RP clinically, and when a fundus pattern is always inherited in the same fashion it is strong evidence supporting a single disease process. However, subsequent research will have to demonstrate which morphological types consistently have the same aetiology, since difference aetiologies may give rise to the same clinical appearance. Such is the case in fundus flavimaculatus, in which autosomal recessive and dominant pedigrees have been reported, ${ }^{8}$ thus suggesting that at least 2 different pathological mechanisms are taking place.

The purpose of this paper is to report a distinctive pattern in $5 \mathrm{RP}$ patients with probable autosomal recessive inheritance. These patients, with advanced disease, have areas of preservation of the retina Correspondence to John R. Heckenlively, MD. pigment epithelium (RPE) adjacent to and under the retinal arterioles, while there is a general loss of the RPE throughout the retina. The amount of preserved para-arteriole retinal pigment epithelium (PPRPE) varies, but it is seen most consistently in the equator and peripheral regions of the eye.

While preservation of macular RPE is commonly seen in advanced RP, this is the first time that a specific morphological pattern of RPE preservation adjacent to retinal arterioles has been reported in retinitis pigmentosa. Since 3 of the 5 patients have pedigrees consistent with autosomal recessive inheritance, and all are hypermetropic, it is likely that these PPRPE patients represent a specific form of RP.

\section{Case reports}

\section{CASE 1}

This is a 9-year-old white boy who failed his vision test in school. There was no family history of ocular problems, and consanguinity was denied. On examination his visual acuity was noted to be RE 20/30, LE 20/40. Cycloplegic refraction was RE $+6 \cdot 00$ $+1 \cdot 00 \times 75, \mathrm{LE}+6 \cdot 00+1 \cdot 00 \times 105$.

Except for a fine nystagmus the external and slitlamp examinations were within normal limits. Fundus examination revealed pale optic discs, with moderate attenuation of the retinal vasculature.

In the areas just anterior the vascular arcades preservation of the RPE could be seen adjacent to and under the retinal arterioles (Fig. 1). This pattern was not consistent throughout the eye and appeared to be 


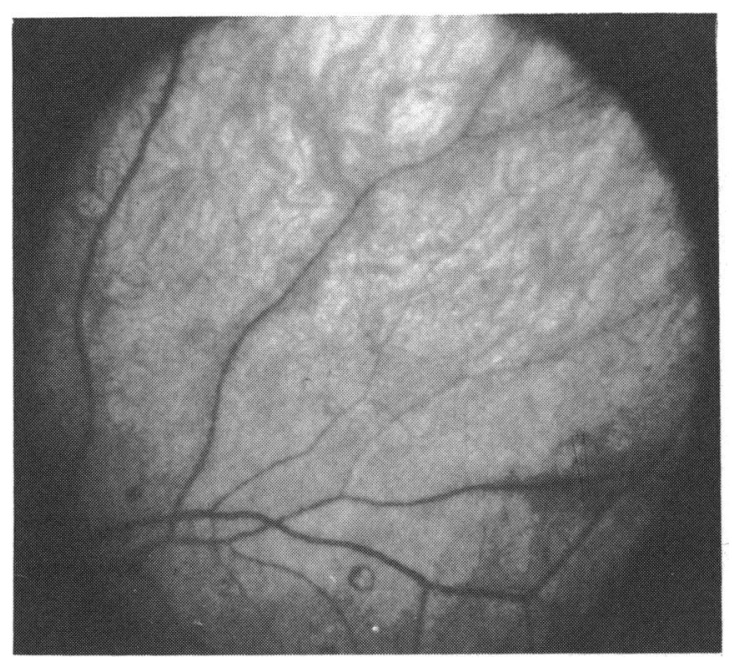

Fig. 1 9-year-old white boy with advanced retinitis pigmentosa, showing preservation of the retinal pigment epithelium adjacent to and under retinal arteriole; left eye.

a relative preservation. On careful review of fundus photographs patchy preservation of the RPE could be seen adjacent to smaller arterioles in the posterior pole. Round clumps of brownish-black pigment were present throughout the eye, which on stereoscopic examination were subretinal or within layers of the retina. Small yellow surface focal deposits were scattered throughout both retinas. Optic nervehead drusen were present in both eyes.

The electroretinogram performed at the referring hospital was reported to be abnormal. The darkadaptation test with a $1^{\circ}$ test object at $11^{\circ}$ above fixation showed a $4 \cdot 6 \log$ unit elevation of the final rod threshold at $\mathbf{4 0}$ minutes. The cone threshold was also elevated, and the rod-cone break was at 17 minutes. The electro-oculogram (EOG) was abnormal, with a light-dark ratio of $100 \%$ in both eyes. The Goldmann visual field with the IV-4 isoptre was RE $30^{\circ}$, LE $15^{\circ}$.

The Farnsworth 100-hue discrimination test had a total error score of RE 191 with an early tritan axis, and LE 261 with a bipolar tritan axis. The Nagel anomaloscope had a Rayleigh equation of RE 42-50, LE 48-52, which is in the protanomalous range.

Peripheral blood smears, phytanic acid levels, vitamin A and carotene levels, quantitative haemoglobin electrophoresis, quantitative amino acids, and serology testing were all reported to be within normal limits.

\section{CASE 2}

This is a 33-year-old Iranian woman who noted night blindness at age 5 and loss of central vision at 17 . The

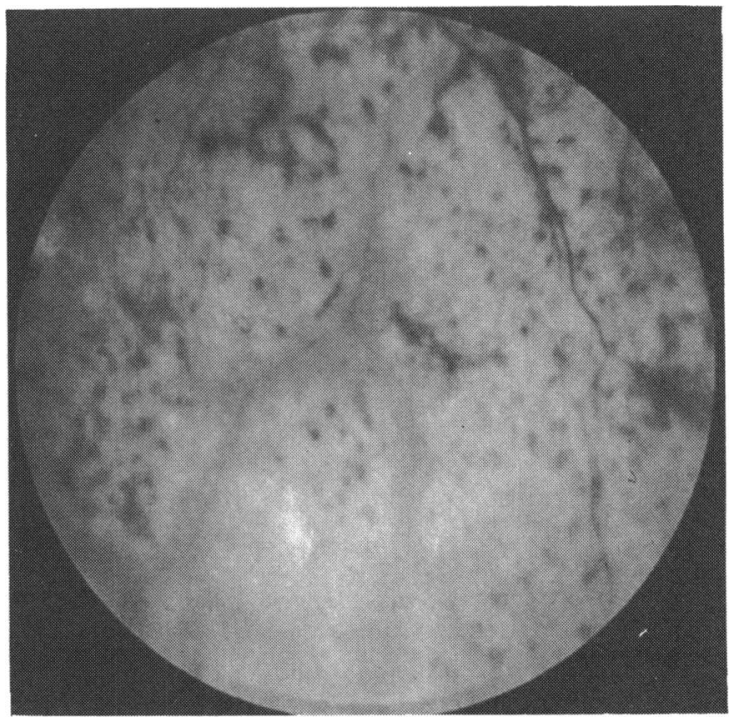

Fig. 2 33-year-old Iranian woman with advanced retinitis pigmentosa with retinal pigment epithelium preservation adjacent to and under equator retinal arteriole. More posterior areas showed no significant preservation of retinal pigment epithelium near arterioles.

medical history was negative. The family history revealed no other member with RP, but the parents were first cousins.

On examination the patient's best corrected visual acuity was RE counting fingers, LE $4 / 200$, with a cycloplegic refraction of $\mathrm{RE}+3 \cdot 00+0 \cdot 75 \times 120, \mathrm{LE}$ $+2 \cdot 50$. Applanation tensions and external examination were normal. Slit-lamp examination revealed bilateral posterior subcapsular cataracts. On fundus examination the optic nerve was pale, and round yellow excrescences could be seen within the nervehead substance. There was a diffuse loss of the RPE with scattered clumps of pigment at the level of the RPE. The retinal vasculature was moderately attenuated.

Preservation of the RPE could be seen next to retinal arterioles in the peripheral regions of both eyes (Fig. 2). There were localised regions where the pattern was definite, and other areas where the RPE was missing next to the arterioles. The pattern was enhanced by fluorescein angiography (Fig. 3).

An electroretinogram (ERG) was nonrecordable, and visual field study was not possible because of the advanced stage of disease. A complete blood count, fluorescent treponemal antibody test, haemoglobin electrophoresis, protein electrophoresis, quantitative amino acids, metabolic urine screen, and haemoglobin oxygenation curve were normal. 


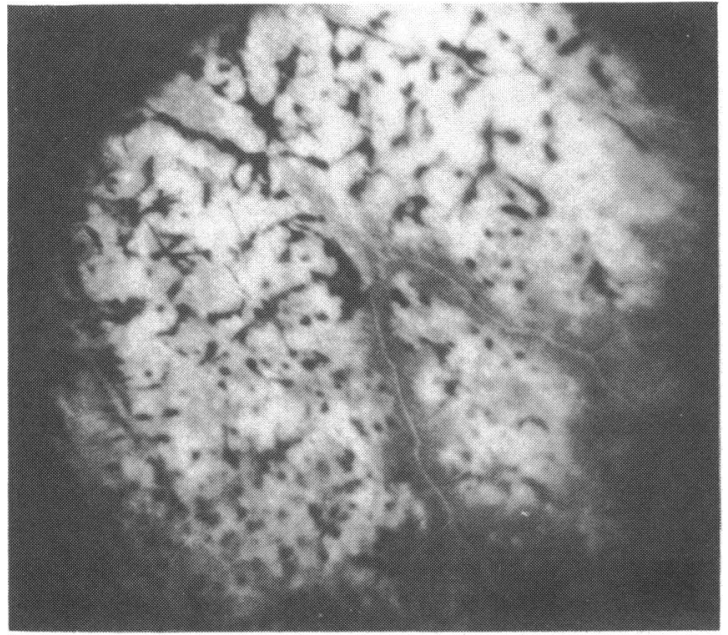

Fig. 3 Fluorescein angiogram, case 2, confirms that the para-arteriole retinal pigment epithelium is preserved, while other retinal areas are hyperfluorescent where the retinal pigment epithelium is functionally lost.

\section{CASE 3}

This is an 18-year-old Mexican woman with a 10-year history of nightblindness. The mother's aunt has been blind since aged 23 from unknown causes. No other family members are reported to have ocular problems, and the medical history is negative with the exception of some psychiatric problems. The patient's visual acuity was RE $20 / 60$ with $+2 \cdot 00+2 \cdot 25 \times 90$, LE $20 / 50$ with $+1 \cdot 50+2 \cdot 75 \times 95$. The patient had a left exotropia of 40 prism dioptres and a fine-jerk nystagmus. The right lens had a small posterior subcapsular cataract, and each eye had $+2 / 4$ pigment in the anterior vitreous.

Fundus examination revealed diffuse loss of the retinal pigment epithelium except for bilateral islands of RPE in the maculae. There was attenuation of the retinal vasculature and scattered areas of pigment deposition, with tiny drusen throughout the fundus. Optic nervehead drusen were seen on stereoscopic examination of fundus photographs. In the right eye 2 areas of distinctly preserved para-arteriole retinal pigment epithelium could be seen in the anterior retina superior temporally and inferior nasally. Six months later the areas of RPE preservation were definitely smaller.

The photopic and scotopic electroretinograms were nonrecordable, and the final rod threshold was elevated by $3.25 \mathrm{log}$ units. The Goldmann visual field with the IV -4 isoptre showed $3^{\circ}$ centrally in each eye. A complete blood count, fluorescent treponemal antibody test, metabolic screen, and quantitative amino acids were normal.

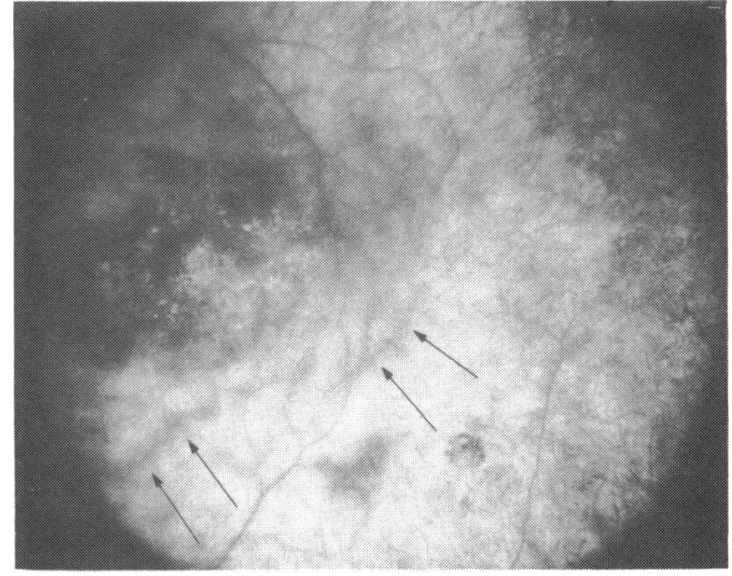

Fig. 4 Fluorescein angiogram of 65-year-old woman with advanced $R P$, showing preservation of the RPE under arteriole coursing temporally inferior to macula (arrows); left eye. This pattern was not apparent on fundus examination, and it was necessary to use fluorescein angiography to confirm the PPRPE pattern.

\section{CASE 4}

This is a 65-year-old white female who noted night blindness by age 30 and stopped driving by age 45 . The family history revealed a great-grandmother who went blind, a sister, now deceased, who had RP, and a first cousin once removed who is blind. There was no history of consanguinity in the pedigree.

On examination the patient's best corrected visual acuity was RE 20/200, LE 20/400, with a correction of $\mathrm{RE}+3 \cdot 75$ and $\mathrm{LE}+2 \cdot 75$. Slit-lamp examination revealed small posterior subcapsular cataracts in each eye. On indirect ophthalmoscopy the patient had a fundus of blond appearance, with vessel attenuation, optic atrophy, and scattered bone spicules and aggregations of pigment in the equator region. The blond-appearing fundus did not allow for the identification of the PPRPE pattern, and a fluorescein angiogram was obtained to look for the preservation of the RPE under the arterioles. Fig. 4 shows the posterior pole in which a small arteriole coursing temporally inferior to the macula shows the PPRPE pattern.

Visual physiological studies demonstrated a nonrecordable photopic and scotopic ERG with a 5.0 log unit elevation of the final rod threshold on dark adaptometry study. The patient had a $4^{\circ}$ Goldmann visual field with a IV-4 isoptre in each eye.

\section{CASE 5}

This is a 15-year-old white boy who was noted to have night blindness and nystagmus at age 15 months. 


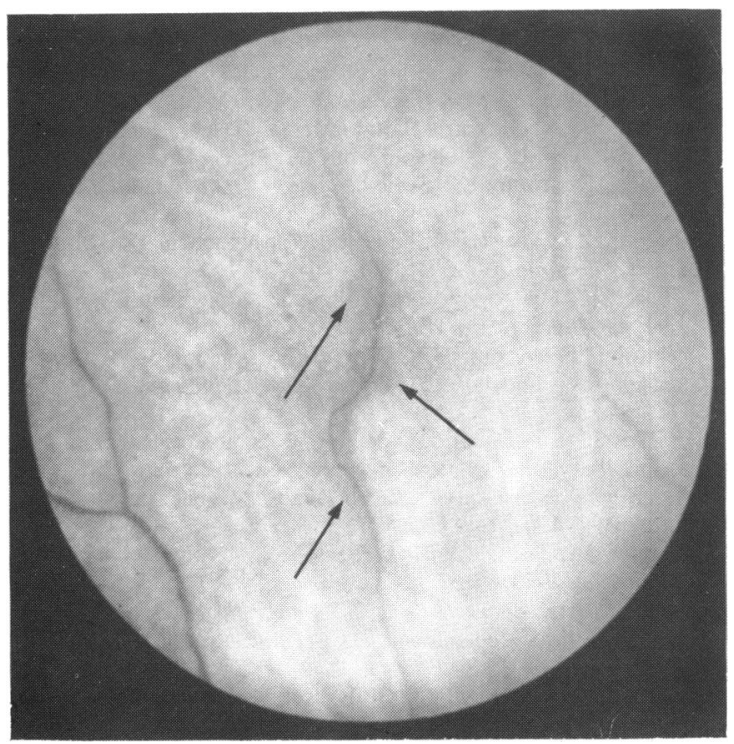

Fig. 5 Fundus photograph of 15-year-old boy with PPRPE in all quadrants of each eye. Para-arteriole retinal pigment epithelium is preserved in anterior equator regions (arrows) despite extensive panretinal degeneration elsewhere.

There is no family history of eye problems or consanguinity. Fundus examination at the time was reported as normal. Subsequent examinations at age 9 revealed mild pigmentary deposits in both eyes, and the diagnosis of retinitis pigmentosa was suspected.

On a recent examination at age 15 the patient had a visual acuity of RE 20/200, LE 20/400, with a correction of $\mathrm{RE}+3.00+0.75 \times 90$, $\mathrm{LE}+2.50$ $+0.75 \times 90$. The patient had a rotary and jerk nystagmus. Slit-lamp examination revealed anterior and posterior subcapsular opacities in both eyes. Fundus examination revealed a blond fundus with generalised diffuse loss of the retinal pigment epithelium, optic nervehead drusen, and a few scattered pigment deposits in the posterior pole. Preserved para-arteriole retinal pigment epithelium could be seen in all quadrants of both eyes in the anterior equator region. Visual physiological testing demonstrated a nonrecordable ERG and a final rod threshold that was $4 \cdot 15 \log$ units elevated at 40 minutes. Goldmann visual fields with the IV-4 isoptre showed $7^{\circ}$ showed central fields with temporal peripheral islands in each eye.

\section{Discussion}

It is important to review those features which support the view that the same disease process is taking place in these patients. In addition to the distinctive PPRPE pattern the patients had other similar appearances in the fundus. All 5 had fine round yellow retinal deposits in the posterior pole. The pigment was clumped in atypical irregular aggregations; and, while some vessels were coated with pigment, there was less generalised bone spicule formation ${ }^{10}$ than is frequently seen in RP. The retinal blood vessels were not as attenuated as is frequently seen in advanced RP, and optic nervehead drusen were seen in 4 cases, with optic atrophy readily apparent in all cases.

While all these findings with the exception of the PPRPE pattern are seen in RP patients, they all occur less often in RP as a group. Optic nerve 'abnormalities' were noted only $65 \%$ of the time by Merin and Auerbach," "and in a recent survey of RP fundus photographs we found that $76 \%$ of RP patients have pink though not necessarily normal-appearing discs. ${ }^{12}$ Other evidence supporting a single disease process is that all the patients were hypermetropic when RP patients as a group are myopic, ${ }^{13} 4$ of the 5 had optic nervehead drusen, and all 5 patients have pedigrees consistent with autosomal recessive inheritance.

The fact that these 5 cases showed focal preservation of the retinal pigment epithelium in areas adjacent to arterioles, despite a panretinal degenerative process, implies some type of positive interaction between the retinal pigment epithelium and the arteriole. In fact advanced disease is necessary to see the pattern of preservation.

Initially it might be assumed that an oxygenation effect from the arteriole helps to preserve the RPE. This does not appear to be likely, since the RPE does not derive its nutrition and oxygen from the retinal circulation. Furthermore any oxygenation effect is just as likely to come from the retinal capillary network as from the retinal arteriole.

If the effect was one of reducing light exposure by the shadow of the overlying vessel, then the same pattern would be expected under and adjacent to the veins. Examination of the choroidal vasculature on fundus photographs and fluorescein angiograms reveals no abnormalities of the choroidal circulation which would help to explain this phenomenon.

It is conceivable that a permeable factor is being released by the retinal arterioles which has a positive influence on the RP process, thereby temporarily retarding the degeneration locally in the areas of the retinal arteriole. If the primary aetiological process in these patients is in the bipolar layer, which receives nourishment from the retinal circulation, the retrograde degeneration ${ }^{14}$ could cause loss of the receptors and RPE except in the proximity of arterioles. 
Likewise transsynaptic degeneration would explain the optic atrophy seen in these patients. It is also possible that a permeable factor could reach the receptors of RPE directly.

Proving the existence of such a factor may be quite difficult, since the aetiology of the disease process is not known. However, understanding the role of the retinal arteriole in PPRPE would contribute significantly to determining the aetiology and pathogenesis of these patients' RP. The PPRPE pattern is not common; the frequency in our RP population has been less than $1 \%$.

Preservation of posterior pole RPE is a common occurrence in RP; the central RPE will remain unchanged and functioning in many patients for a number of years. The reasons for the macular RPE being resistant to the disease are not known, but physiological differences between central and peripheral RPE are beginning to be reported ${ }^{15}$ and eventually may clarify this mystery.

Retinitis pigmentosa is a nonspecific diagnosis, typically indicating a pigmentary retinopathy, nonrecordable or abnormal electroretinogram, and progressive visual field loss. However, because RP occurs in so many diseases and inheritance patterns, classification systems by aetiology or at least pathogenesis need to be developed. This will allow research efforts to be focused more effectively, and will aid the practitioner in more precisely diagnosing the various types of RP.

Dr Allan Kreiger. Dr William Stell, and Dr David Cogan reviewed the cases. Dr F. D. Ellis referred case 1. Jean Shimizu and Mada Barrett provided secretarial and editorial assistance.

\section{References}

1 Best F. Uber eine hereditare maculaaffektion. Beitrage zur Vererbungslehre. Arch Augenheilkd 1905; 13: 199-212.

2 Deutman AF, Van Blommestein JDA, Henkes HE, Waardenburg PJ, Solleveld-Van Driest E. Butterfly-shaped pigment dystrophy of the fovea. Arch Ophthalmol 1970; 83: 558-69.

3 O'Donnell F, Welch RB. Fenestrated sheen macular dystrophy. Arch Ophthalmol 1979; 97: 1292-6.

4 Sjogren H. Dystrophia reticularis laminae pigmentosae retina. Acta Ophthalmol 1950; 28: 279-95.

5 Pearlman JT, Kamin DF, Kopelow SM, Saxton J. Pigmented paravenous retinochoroidal atrophy. Am J Ophthalmol 1975; 80: $630-5$.

6 Franceschetti A. A curious affection of the fundus oculi: helicoid peripapillary chorioretinal degeneration. Its relation to pigmentary paravenous chorioretinal degeneration. Doc Ophthalmol 1962; 16: 81-110.

7 Krill AE, Archer D, Martin D. Sector retinitis pigmentosa. Am J Ophthalmol 1970; 69: 977-87.

8 Krill AE. Hereditary Retinal and Choroidal Diseases: Clinical Characteristics. Hagerstown: Harper and Row, 1977: 2: 500, 747-8.

9 Pearlman JT, Flood TP, Seiff SR. Retinitis pigmentosa without pigment. Am J Ophthalmol 1976; 81: 417-9.

10 Meyer K, Heckenlively JR, Foos RY, Spitzas M. Clinicopathologic correlation in dominant retinitis pigmentosa. Submitted for publication.

11 Merin S, Auerbach E. Retinitis pigmentosa. Surv Ophthalmol 1976; 20: 303-46.

12 Rajacich GM, Heckenlively JR, Meyer K, Cavender JC. The optic nervehead in retinitis pigmentosa. Invest Ophthalmol Visual Sci 1981; suppl ARVO.

13 Sieving PA, Fishman GA. Refractive errors of retinitis pigmentosa patients. Br J Ophthalmol 1978; 62: 163-7.

14 Gills JP, Wadsworth JAC. Retrograde transsynpatic degeneration of the inner nuclear layer of the retina. Invest Ophthalmol Visual Sci 1967; 6: 437-48.

15 Newsome DA, Fletcher RT, Chader GJ. Cyclic nucleotides vary by area in retina and pigmented epithelium of human and monkey. Invest Ophthalmol Visual Sci 1980; 19: 864-9. 